Ensino, Saúde e Ambiente - V9 (3), pp. 297-309, Dez. 2016

\title{
A QUESTÃO AMBIENTAL E A ENFERMAGEM: PERCEPÇÕES DE ENFERMEIROS E ESTUDANTES
}

\section{THE ENVIRONMENTAL ISSUE AND NURSING: PERCEPTIONS OF NURSES AND STUDENTS}

\author{
Andreia Aparecida Guimarães Strohschoen ${ }^{1}$, Claudete Moreschi ${ }^{2}$, Claudete Rempel $^{3}$ \\ ${ }^{1}$ Centro Universitário UNIVATES/Centro de Ciências Biológicas e da Saúde/ Programa de Pós- \\ Graduação em Ensino, aaguim@univates.br \\ ${ }^{2}$ Centro Universitário UNIVATES/Programa de Pós-Graduação em Ambiente e Desenvolvimento, \\ clau_moreschi@yahoo.com.br \\ ${ }^{3}$ Centro Universitário UNIVATES/Centro de Ciências Biológicas e da Saúde/Programas de Pós- \\ Graduação em Ambiente e Desenvolvimento e Sistemas Ambientais Sustentáveis, crempel@univates.br
}

\section{RESUMO}

O presente estudo visou analisar as percepções de enfermeiros e estudantes de enfermagem acerca da questão ambiental. A pesquisa é descritivo-exploratória, de caráter qualiquantitativa e foi realizada por meio da aplicação de questionários, com questões objetivas e descritivas, a 25 enfermeiros atuantes na rede de saúde de seis municípios do estado do Rio Grande do Sul e 30 estudantes de diferentes semestres do curso de graduação em Enfermagem de uma Instituição de Ensino Superior. Os questionários foram aplicados no período de agosto a novembro de 2015. Verificou-se que a temática ambiental estava sendo abordada durante a formação acadêmica dos enfermeiros em uma disciplina do primeiro semestre. O tema "resíduos de serviços de saúde" foi o mais citado, seguido em ordem decrescente os seguintes assuntos: vigilância sanitária, meio ambiente, ecologia, problemas ambientais e saneamento básico. Os alunos, ao conceituarem "Meio Ambiente", demonstraram a percepção de que este se constitui como eminentemente físico. Dentre as atribuições do enfermeiro frente às questões ambientais, emergiu a percepção de que este profissional deve envolver-se com ações educativas relacionadas à educação ambiental com vistas à conscientização da população. Sobre as ações de cuidado com o meio ambiente, emergiu que os participantes procuram reduzir a produção de resíduos, destiná-los adequadamente e reduzir o consumo da energia elétrica e água.

Palavras-chave: meio ambiente, enfermagem, ensino

\begin{abstract}
This paper aimed to analyze the perceptions of nurses and nursing students concerning environmental issue. The research is descriptive-exploratory, with a qualitative and quantitative approach and was performed through applied questionnaires, with closed questions, to 25 nurses working in the health network system of six municipalities from Rio Grande do Sul state and 30 students, from different study levels, in a University graduate Nursing course The questionnaires were applied between August and November 2015. It was verified that the environmental theme was being worked during the first semester nurse academic training the theme "waste health services" was the most cited, followed by these subjects in descending order: health surveillance, environment, ecology, environmental problems and basic sanitation. The students, when conceptualized "Environment", demonstrated the perception that this was constituted as eminently physical. Among the nurse's attributions regarding environmental issues, it
\end{abstract}


emerged that this professional should get involved in educational activities related to environmental education, aiming to aware the population. Regarding environment care actions, it emerged that the participants were trying to reduce waste production, to dispose them properly and to reduce the electricity and water consumption.

Key words: environment, nursing, education

\section{INTRODUÇÃO}

A Organização Mundial da Saúde apresenta o conceito de meio ambiente como sendo tudo que é externo ao homem, sendo físico, biológico, social e cultural. Ressalta que qualquer um ou todos podem interferir no estado de saúde da população (OMS, 1995). A ampliação da responsabilidade com o meio ambiente no contexto da saúde busca compreender a questão ambiental como um evento complexo, socialmente construído. Tal atitude requer empenhos individuais e coletivos para sua resolubilidade, tendo importante interferência sobre o processo saúde-doença (CAMPONOGARA et al., 2013).

O enfoque integrado acerca das questões envolventes sobre saúde e ambiente é decorrente do final dos anos 80, quando ambientalistas e sanitaristas, investigadores e gestores iniciaram a percepção da necessidade de articulação entre teoria e ação com a ideia da qualidade de vida de grupos populacionais. Esse propósito emergiu a partir da convicção de que para existir desenvolvimento sustentável é necessário considerar os seres humanos e sua vida no ecossistema (GOMES; MINAYO, 2006).

Na I Conferência Internacional sobre Promoção da Saúde, em 1986, foi constituída a Carta de Ottawa, a qual designou em um de seus enfoques de ação, a concepção de ambientes adequados à saúde (BRASIL, 1986). Essa área que trata da inter-relação entre saúde e meio ambiente foi denominada de Saúde Ambiental. O Ministério da Saúde define a saúde ambiental como sendo área da saúde pública que afeta ao conhecimento científico e à formulação de políticas públicas relacionadas à interação entre a saúde humana e os fatores do meio ambiente natural e antrópico que a determinam, condicionam e influenciam, com vistas a melhorar a qualidade de vida do ser humano, sob o ponto de vista da sustentabilidade (BRASIL, 2005).

Ao considerar que uma vida saudável está relacionada diretamente a um planeta saudável, existe uma preocupação crescente com o meio ambiente nas diferentes áreas do conhecimento (RIBEIRO; BERTOLOZZI, 2002). Trata-se, portanto, de uma vasta área de estudo, que contempla profissionais de diferentes formações acadêmicas e técnicas (RIBEIRO; BERTOLOZZI, 2004). Na enfermagem, essa inquietação existe 
desde os primórdios da profissão como ciência, tendo início com a Teoria Ambientalista proposta pela precursora da enfermagem Florence Nightingale (MORESCHI et al., 2011).

Florence Nightingle recomendou atenção ao ambiente na prestação da assistência de enfermagem, mas, mesmo assim, há poucos estudos que tratam este assunto considerando a vertente ecológica (RIBEIRO; BERTOLOZZI, 2004). Para ela o cuidado com o ambiente tinha por base a corrente Higienista e o conceito de ambiente restringia-se ao espaço físico onde ficava o doente e a família. Observa-se que mesmo 150 anos depois da Florence, o conceito de ambiente, nos trabalhos de enfermagem, quase não mudou (RIBEIRO; BERTOLOZZI, 2002).

A interface saúde e meio ambiente precisa ser incorporada nos debates em saúde, devido ser uma necessidade contemporânea que requer do setor saúde a construção de bases teóricas e práticas responsáveis com a preservação do planeta para essa e para as futuras gerações (CAMPONOGARA et al., 2013). No processo de ensino dos estudantes de enfermagem, é relevante intensificar debates acerca da temática saúde e meio ambiente, pois enquanto profissionais terão atuações em atividades de promoção e reabilitação em saúde. Tais campos poderão abrir possibilidades para que os profissionais possam desenvolver práticas voltadas para uma educação ambiental, enfatizando a importância de um despertar de sujeitos ecológicos (CAMPONOGARA et al., 2011). Assim, pressupõe que o cuidado de enfermagem necessita contemplar o ser humano em todas as suas relações e interações com o meio ambiente (MORESCHI et al., 2011).

Por esses motivos, é necessário realizar estudos sobre como os profissionais da enfermagem atuam frente às questões relacionadas com a área ambiental. Desta forma, o presente estudo teve por objetivo analisar as percepções de enfermeiros e estudantes de enfermagem acerca da questão ambiental.

\section{MATERIAL E MÉTODOS}

A pesquisa é descritivo-exploratória, de caráter qualiquantitativa e foi realizada por meio da aplicação de questionários, com questões fechadas, a 25 enfermeiros atuantes na rede de saúde de seis municípios do estado do Rio Grande do Sul e 30 estudantes de diferentes semestres do curso de graduação em Enfermagem de uma 
Instituição de Ensino Superior (IES) também do Rio Grande do Sul. Os questionários foram aplicados no período de agosto a novembro de 2015.

Inicialmente foram convidados a participar da pesquisa alunos matriculados no curso de Enfermagem de uma IES comunitária do interior do Rio Grande do Sul. O convite foi realizado em aulas de diferentes disciplinas do referido curso, de forma aleatória. Além dos alunos, enfermeiros de seis municípios da região de abrangência da IES foram convidados, por meio de correio eletrônico, para participar da pesquisa. Retornaram 25 aceites de profissionais oriundos dos municípios: Arroio do Meio, Estrela, Lajeado, Marques de Souza, Venâncio Aires e Santa Cruz do Sul e 30 aceites dos alunos de Enfermagem.

Foram aplicados questionários semiestruturados compostos por questões objetivas e descritivas. As questões versavam sobre quais assuntos relacionados à questão ambiental foram abordadas durante a formação no curso de graduação em Enfermagem; conceito de Meio Ambiente; papel do enfermeiro frente às questões ambientais; atitudes que têm tomado em relação aos cuidados com o ambiente e cuidados necessários em relação aos resíduos de serviços de saúde.

Os dados obtidos a partir das questões objetivas foram analisados através de estatística descritiva e inferencial, sendo as frequências das respostas das questões objetivas apresentadas em forma de percentual (\%). As questões subjetivas foram analisadas por meio da Análise Descritiva (PEREIRA, 1999). Para a questão que trata do cuidado com os resíduos de serviços de saúde foi utilizada a Análise de Conteúdo (BARDIN, 2011).

Com a finalidade de compreender os dados, foi realizada a análise do Projeto Pedagógico do curso de Enfermagem dos estudantes participantes do estudo. Após captura dos documentos disponíveis na página online do curso, foi realizada uma leitura minuciosa na matriz curricular do curso referido.

As questões éticas foram respeitadas conforme as recomendações da Resolução n. 466/12 do Conselho Nacional de Saúde sobre Pesquisas com Seres Humanos (BRASIL, 2012). O estudo foi aprovado pelo Comitê de Ética em Pesquisa do Centro Universitário UNIVATES, de Lajeado/RS, sob o $\mathrm{n}^{\circ}$. 16660. Os estudantes e enfermeiros que aceitaram participar da pesquisa, responderam individualmente ao questionário após assinatura do Termo de Consentimento Livre Esclarecido (TCLE). Para manter o sigilo 
quanto às suas identidades e suas falas, os participantes estudantes da pesquisa foram identificados pela letra "E" e os profissionais enfermeiros pela letra "P".

\section{RESULTADOS E DISCUSSÃO}

O grupo estudado foi formado por 55 indivíduos, sendo 25 enfermeiros atuantes na rede de saúde e 30 estudantes do curso de graduação em Enfermagem, sendo cinco estudantes do segundo semestre, quatro do terceiro, três do quarto, quatro do quinto, dois do sexto, dois do sétimo, seis do oitavo e quatro estudantes do nono semestre. Observa-se assim, que há estudantes do segundo ao nono semestre do curso, fornecendo um panorama da graduação.

Mulheres compunham $84 \%$ do total de participantes deste estudo. Nos cursos de enfermagem é comum observarmos a maior presença do sexo feminino. Na enfermagem brasileira, ainda existe a feminização, sendo que isto pode, principalmente, ser observado na qualificação universitária e nos níveis médio e técnico (LOPES; LEAL, 2005).

Em relação à atuação dos enfermeiros participantes desta pesquisa, 19 (76\%) atuam em hospitais da região, quatro (16\%) atuam em Unidades Básicas de Saúde (UBS) ou Estratégias de Saúde da Família (ESF), dois relataram estarem vinculados atualmente com o Ensino, atuando como professores de cursos de graduação. Quanto aos estudantes observam-se que oito (27\%) já atuam em hospitais, os demais estão atualmente vinculados a diferentes atividades: assistente de vendas, atendente de farmácia, mercearias entre outros.

Os participantes relataram os seguintes assuntos como tendo sido abordados durante o curso de graduação em enfermagem: Resíduos de Serviços de Saúde - RSS sendo citado por 41 dos participantes (74\%), seguido do tema vigilância sanitária, citado por 32 participantes (58\%), meio ambiente citado por 29 participantes (53\%), ecologia citado por 24 (44\%), problemas ambientais, citado por 21 (38\%) e saneamento básico, citado por 20 (36\%) dos participantes. Cabe salientar que estes podiam citar até três temas relacionados à questão ambiental.

Quanto aos temas abordados durante a graduação em enfermagem acerca da questão ambiental, observa-se que a temática dos RSS foi o assunto que apresentou maior prevalência. Outro estudo, realizado na mesma região do presente, evidenciou que o conhecimento sobre a temática dos RSS foi abordada superficialmente durante a 
formação acadêmica dos profisssionais da área da saúde. Durante a graduação, constatou-se ausência ou fragmentação de abordagem sobre RSS, evidenciando que esta temática é pouco trabalhada na formação acadêmica dos cursos de graduação da área da saúde (MORESCHI et al., 2014a). O estudo de Bataglin, Souza e Camponogara (2012) também constatou que os trabalhadores de enfermagem, ainda carecem de informações sobre a questão ambienta, em especial à temática sobre RSS e expressam certo desconhecimento sobre o processo de gerenciamento de resíduos. Ao mesmo tempo, informam desconhecer o planejamento da instituição sobre o gerenciamento de resíduos.

Ao analisar as disciplinas do curso de enfermagem verificou-se que a temática ambiental é abordada em uma disciplina do primeiro semestre denominada "Ecologia, Evolução e Saúde Humana" com carga horária de 30 horas. Esta apresenta na ementa a seguinte descrição: "Principais teorias evolutivas e suas implicações na concepção da saúde humana". Além desta disciplina, há a disciplina eletiva: "Environment and Health Issues". Esta apresenta como ementa: "Interdisciplinary themes in the relationship between humans, health and the environment" (que pode ser traduzido por "Temas interdisciplinares na relação entre os seres humanos, saúde e meio ambiente"). É uma disciplina totalmente em língua inglesa, sendo oferecida a vários cursos de graduação da IES em estudo.

Nos currículos dos cursos de graduação na área da saúde, é importante a inclusão do tema da questão ambiental para promover uma postura de responsabilidade socioambiental, por parte dos futuros enfermeiros (CAMPONOGARA et al., 2013). Além disso, é necessário conscientizar os estudantes acerca das questões ambientais e suas consequências para os seres vivos para que estes estabeleçam ações concretas visando o enfrentamento dessas questões (GONZÁLEZ-GAUDIANO; LORENZETTI, 2009). Cunha e Valente (2009) concluíram que alguns fatores influenciam mais o conhecimento dos trabalhadores de enfermagem sobre as questões ambientais, como o interesse pessoal ou estímulo, falta de tempo, sobrecarga de trabalho, treinamentos inadequados, disponibilidade de EPIs e condições de trabalho.

Assim, entende-se que é necessário ampliar as discussões sobre a questão ambiental nos cenários do ensino e do trabalho, no campo da enfermagem e de toda a saúde. Para Camponogara et al. (2012) o que se propõe é uma reflexão aprofundada, que instigue os sujeitos a refletir essa temática com ética, na expectativa de alcançar a responsabilização com a causa ambiental . Nos currículos de formação dos profissionais 
de saúde é preciso construir práticas pedagógicas que permitam a compreensão da questão ambiental como um processo a ser construído, no decorrer do ensino (GONZÁLEZ-GAUDIANO; LORENZETTI, 2009). Para Bataglin, Souza e Camponogara (2012) as instituições de saúde também devem investir em capacitações sobre o tema, sendo esta, considerada a estratégia ideal para oportunizar uma sensibilização e maior aporte de conhecimentos, aos trabalhadores, traduzindo-se em compromisso institucional com o meio ambiente.

Considerando o conceito do termo Meio Ambiente, observaram-se as seguintes respostas: 18 participantes $(33 \%)$ relacionaram este termo aos seres vivos e não vivos que existem no planeta. Não houve menção de que os seres humanos estariam inseridos no meio ambiente. Outra resposta frequente foi fornecida por 37 (67\%) participantes, relacionando o Meio Ambiente como sendo o local onde os seres vivos e não vivos estão inseridos, sendo o local onde vivemos. Nesta resposta foi mencionado que é o local onde o homem vive.

Prevaleceu nas respostas uma percepção de que o meio ambiente se constitui como eminentemente físico, não havendo uma visão ampla, onde o ser humano está inserido, contemplando a relação do homem com a natureza e a sociedade. Esta interpretação de meio ambiente tem sua base no Higienismo, que limitam suas ideias ao saneamento básico. Não havendo relação dos impactos antropogênicos adversos com a degradação do ambiente (RIBEIRO; BERTOLOZZI, 2004).

O Meio ambiente continua sendo visto como algo exterior ao ser humano. É necessário criar e fortalecer estratégias que busquem contribuir com o resgate da relação homem-natureza. Para isso, torna-se imprescindível que o homem possua uma nova compreensão acerca da natureza, deixando de percebê-la apenas como uma matéria prima. O homem pode e precisa visar o desenvolvimento do ambiente pautado na sustentabilidade, não o degradando e não interferindo na qualidade de vida da comunidade local (RODRIGUES; FEIJÓ, 2007).

Quanto ao papel do enfermeiro frente às questões ambientais, foram observadas as seguintes respostas: 24 (34\%) dos participantes relataram que um dos principais papéis do enfermeiro deve estar relacionado à conscientização, norteados por ações educativas relacionadas à educação ambiental da população; 20 (29\%) relataram que o enfermeiro deve principalmente preocupar-se com a separação e descarte correto de lixo e material descartável; 13 (18,5\%) mencionaram a prevenção de doenças relacionadas a problemas ambientais; foram ainda citados por $13(18,5 \%)$ dos 
respondentes que o enfermeiro deve preocupar-se com a redução da produção de resíduos e poluição ambiental. Os participantes apresentaram mais do que uma resposta para a pergunta.

$\mathrm{Na}$ revisão de Ribeiro e Bertolozzi (2002) observa-se que a grande preocupação dos enfermeiros, considerando a questão ambiental, relaciona-se com a produção de resíduos e seu descarte, confirmando nossos achados. Todavia, estudo que objetivou conhecer a percepção de docentes de cursos de graduação da área da saúde acerca dos RSS, constatou-se que, muitas vezes, os profissionais que manipulam os RSS não se preocupam com a produção, os destinam de forma inapropriada ou meramente aceitam a simples informação de que precisam segregar adequadamente tais resíduos, se despolitizando enquanto cidadãos consumidores. É importante promover a conscientização desses profissionais para se tornarem consumidores responsáveis pelos RSS no decorrer de suas práticas (MORESCHI; REMPEL; BACKES, 2014).

Os indivíduos, enquanto cidadãos, precisam sentir-se responsáveis em proteger o meio ambiente contra a degradação, no intuito de diminuir os agravos provenientes das ações antrópicas para as futuras gerações. Cabe aos profissionais de saúde refletir sobre a importância do cuidado ecológico em sua amplitude e complexidade. No decorrer de suas práticas do processo de assistência à saúde das populações, é importante que os profissionais busquem mais informações para agir de maneira consciente e responsável com vistas à preservação ambiental e à valorização dos determinantes ambientais (CAMPONOGARA et al., 2012).

Os participantes, ao serem questionados sobre quais as atitudes que eles têm tomado atualmente em relação aos cuidados com o ambiente, listaram que procuram destinar os resíduos nos locais adequados, preocupando-se com a separação e destinação dos resíduos (42 - 65\%); sete $(11 \%)$ relatam que procuram reduzir a produção de resíduos; Também sete (11\%) buscam reduzir o consumo de água, energia elétrica, papel, dentre outros; cinco $(7,5 \%)$ atuam na orientação das equipes de trabalho onde estão inseridos e quatro (5,5\%) têm atuado na orientação da população quanto à preocupação com as questões ambientais. Os participantes apresentaram mais do que uma resposta para a pergunta.

Quanto aos cuidados em relação aos resíduos de serviços de saúde, os estudantes relataram a importância da segregação adequada, ao uso de EPIs pelas pessoas que manuseiam os resíduos, ao descarte apropriado, a importância de evitar a contaminação e também acreditam que é importante ao profissional saber orientar as 
pessoas sobre o manejo apropriado dos resíduos. Entendem que é necessário segregar de maneia adequada para evitar a contaminação de outros resíduos como podem ser vistos nas falas dos Estudantes E1, E3, E4, E7, E8 e E10 descritas a seguir.

Todos os cuidados possíveis para separação de resíduos e consequentemente o seu descarte correto, contribuindo assim para prevenção de doenças (E1).

Separar ele de maneira correta, pois existem resíduos que podem contaminar outros resíduos e pessoas, e dar o destino certo (E3).

Separação correta de cada um dos resíduos, contaminantes, infectantes, sólidos, líquidos e o uso de EPIS ao manuseio dos resíduos (E4).

Saber onde eles serão descartados, para que não seja jogado diretamente no ambiente (E7).

Temos os resíduos biológicos, aqueles que sem o manuseio correto pode contaminar a si e a outros uso de EPI's é essencial para evitar a contaminação (E8).

Deve ter um profissional responsável por esta função, mas toda equipe deve colaborar colocando cada material no seu devido lugar para que não haja contato com material contaminado (E10).

Os estudantes alegam que os profissionais de enfermagem precisam ter conhecimento sobre o local que os resíduos são destinados na última etapa de seu manejo, para que não aconteça destes serem liberados diretamente no meio ambiente. Acreditam que tais cuidados podem prevenir doenças oriundas do manejo impróprio dos resíduos e evitar a contaminação ambiental.

Os enfermeiros, por sua vez, também mencionam alguns cuidados que julgam necessários em relação ao manejo apropriado dos RSS. Os cuidados relatados foram referentes ao uso de EPI's, segregação adequada, identificação e ao descarte final dos resíduos de maneira correta como pode ser observado nas falas de P6, P7, P11, P12 e P13 transcritas a seguir.

Local apropriado para descartar estes resíduos, identificação dos mesmos (P4).

Atentar para a separação, coleta de materiais recicláveis, químicos, contaminados, pérfuro-cortantes (P6).

O profissional deve utilizar EPI(s), saber a destinação dada para cada tipo de material e cobrar medidas quando não estiverem de maneira correta (P7).

Fiscalização do destino final (P11).

Separação correta dos resíduos, controle de gastos, economia de material, destinação correta (P12).

Cuidado com a contaminação pessoal e do ambiente (P13).

Os enfermeiros percebem que é importante aos profissionais possuírem conhecimento sobre o manejo apropriado dos RSS, visando qualificar a tomada de 


\section{Ensino, Saúde e Ambiente - V9 (3), pp. 297-309, Dez. 2016}

decisões diante de irregularidades encontradas. Acreditam ainda que a efetividade de cuidados com o manejo dos RSS pode evitar a contaminação pessoal e do ambiente.

Percebe-se que tanto os estudantes quanto os enfermeiros atentam para a importância da efetividade de cuidados referentes à segregação, descarte e destino corretos dos resíduos. Pontuam que é relevante ao profissional de enfermagem possuir conhecimento e aptidão para orientar aos demais profissionais sobre o manejo adequado dos resíduos. Outro cuidado mencionado pelos estudantes e enfermeiros, foi o uso de EPIs pelos profissionais ao manusearem os resíduos. Além disso, os participantes vislumbram que a concretude de cuidados apropriados com o manejo adequado dos RSS pode evitar a contaminação do meio ambiente.

Um estudo que buscou conhecer a percepção dos docentes, discentes e egressos da área da saúde de duas instituições de ensino superior do RS acerca da geração dos RSS, constatou que os participantes, de modo geral, atribuem significados aos RSS atentando para a importância da segregação adequada e destino final, sobretudo, com os resíduos contaminados, pois consideram que esses podem provocar danos para a sociedade (MORESCHI et al., 2014b).

A prática da segregação e destino correto de todos os RSS merece atenção especial, uma vez que o descarte inadequado dos resíduos pode provocar impasses ambientais e, consequentemente, colocar em risco os recursos naturais e a qualidade de vida das atuais e futuras gerações (BRASIL, 2006; PEREIRA et al., 2013). O descarte inapropriado dos resíduos poderá desencadear repercussões negativas em todos os seres do planeta. É preciso, deste modo, que os profissionais de saúde tenham um olhar no sentido de mútua pertença entre meio ambiente e produção de resíduos (MORESCHI et al., 2014b).

Frente a isso, destaca-se a importância da efetividade do gerenciamento dos RSS nos serviços de saúde, o qual visa minimizar a produção de resíduos e proporcionar a estes um encaminhamento seguro e eficiente, com o intuito de proteção dos trabalhadores, de preservação da saúde pública, dos recursos naturais e do meio ambiente (BRASIL, 2006). Ainda, na conjuntura da problemática do RSS, acredita-se que o conhecimento é a ferramenta imprescindível para pensar em qualquer ação que possa diminuir riscos oriundos de sua produção desnecessária e destino incorreto. Por meio deste, é possível conhecer e refletir sobre o problema, porém, é necessário ter consciência de que o conhecimento é sempre incompleto e que a realidade é mais complexa do que se pensa. É necessário criar a responsabilidade social nos estudantes 
Ensino, Saúde e Ambiente - V9 (3), pp. 297-309, Dez. 2016

acerca da importância de tomar atitudes sustentáveis, isto é, socialmente justa, economicamente viável e ecologicamente correta (MORESCHI et al., 2014a).

Ao discutir sobre as questões ambientais na área da enfermagem, acredita-se que, por meio de uma educação ambiental que contemple a interface saúde e meio ambiente, na formação profissional, seja possível produzir e resgatar junto à enfermagem um ideário ecológico preocupado com essa teia de conexões dinâmicas entre homem e meio ambiente, seres vivos e não vivos. Tal atitude se constitui em uma das táticas importantes para concretizar o envolvimento da enfermagem em ações sociais ambientalmente apropriadas (CAMPONOGARA et al., 2011).

\section{CONCLUSÃO}

O presente estudo possibilitou analisar as percepções de enfermeiros e estudantes de enfermagem acerca da questão ambiental. Na percepção dos participantes, a temática ambiental está sendo abordada durante a formação acadêmica dos enfermeiros, sendo que os RSS representou o tema mais citado, seguido em ordem decrescente os seguintes assuntos: vigilância sanitária, meio ambiente, ecologia, problemas ambientais e saneamento básico. Verificou-se que tal temática é abordada em uma disciplina do primeiro semestre do curso de enfermagem. Ainda, o conceito de Meio Ambiente é percebido com maior prevalência como um meio que se constitui eminentemente físico, não havendo uma visão ampla, onde o ser humano está inserido, ou seja, não contemplando a relação do homem com a natureza e a sociedade. Quanto ao papel do enfermeiro frente às questões ambientais, emergiu que este profissional deve envolver-se com ações educativas relacionadas à educação ambiental com vistas à conscientização da população.

Sobre as atitudes que os enfermeiros e estudantes têm tomado em relação aos cuidados com o ambiente, evidenciou-se que estes procuram destinar os resíduos nos locais adequados; também procuram reduzir a produção de resíduos, o consumo de água, o consumo de energia elétrica e o consumo de papel. Os participantes têm atuado nas orientações das equipes de trabalho onde estão inseridos, bem como da população em geral. Os cuidados necessários em relação aos RSS são atrelados à segregação correta, descarte e destino correto dos resíduos. Além disso, os participantes vislumbram que a concretude de cuidados apropriados com o manejo adequado dos RSS pode evitar a contaminação do meio ambiente. 
Ensino, Saúde e Ambiente - V9 (3), pp. 297-309, Dez. 2016

Conclui-se, portanto, que o presente estudo possibilitou uma reflexão acerca da importância da enfermagem ampliar as discussões a respeito da questão ambiental. A enfermagem como ciência do cuidado, precisa cada vez mais unir forças entre ensino e serviço em prol da questão ambiental, visando contemplar uma relação de mútua pertença entre homem, natureza e sociedade.

\section{REFERÊNCIAS}

BARDIN, L. Análise de conteúdo. 6 ed. Lisboa: Edições 70, 2011.

BATAGLIN, M. S.; SOUZA, N. H. T. de; CAMPONOGARA, S. Conhecimento da equipe de enfermagem sobre a segregação dos resíduos sólidos em ambiente hospitalar. Ensino, Saúde e Ambiente, v. 5, n. 3, p. 69-83, 2012

BRASIL. Ministério da saúde. Conferência Nacional de Saúde, $8^{\text {a }}$. Relatório Final. Brasília, 1986.

BRASIL. Manual de gerenciamento de resíduos de serviços de saúde. Agência Nacional de Vigilância Sanitária. Brasília (DF): Ministério da Saúde, 2006.

BRASIL. Ministério da Saúde. Instrução Normativa $n^{\circ} 01$. Regulamenta a Portaria GM/MS n ${ }^{\circ}$ 1.172/2004 no que se refere às competências da União, estados, municípios e Distrito Federal na área de vigilância em Saúde ambiental. Brasília, 2005.

BRASIL. Ministério da Saúde. Conselho Nacional de Saúde. Resolução nº 466 de 12 de dezembro de 2012. Diretrizes e normas regulamentadoras de pesquisa envolvendo seres humanos. Brasília, 2012.

CAMPONOGARA, S.; VIERO, C. M.; SARI, V.; ERTHAL, G. A abordagem da interface saúde e meio ambiente na formação profissional de enfermeiros. Rev Gaúcha Enferm., v. 32, n. 4, p. 647-53, 2011.

CAMPONOGARA, S.; SOARES, S. G. A.; VIERO, C. M.; ERTHAL, G. DIAZ, P. S.; PERES, R. R.; ROSSATO, G. C. Responsabilidade ambiental na visão de acadêmicos da área da saúde. Rev. enferm., v. 20, n.1, p. 39-44, 2012.

CAMPONOGARA, S.; VIERO, C. M.; ERTHAL, G.; DIAZ, P. S.; ROSSATO, G. C.; SOARES, A. S.; PERES, R. R. Visão de profissionais e estudantes da área de saúde sobre a interface saúde e meio ambiente. Trab.Educ.Saúde, v. 11, n. 1, p. 93-111, 2013.

CUNHA, A. C. da; VALENTE, G. S. C. Desvelando o conhecimento dos trabalhadores de Enfermagem acerca dos riscos biológicos na Emergência. Ensino, Saúde e Ambiente, v. 2, n. 2, p. 69-83, 2009.

GOMES, M. C.; MINAYO, M. C. Enfoque Ecossistêmico De Saúde: Uma Estratégia Transdisciplinar. InterfaceHs, v. 1, 'n. 1, p. 1-19, 2006. 
GONZÁLEZ-GAUDIANO, E.; LORENZETTI, L. Investigação em educação ambiental na América: mapeando tendências. Educação em Revista, v. 25, n. 3, p. 191-211, 2009.

LOPES, M. J. M.; LEAL, S. M. C. A feminização persistente na qualificação profissional da enfermagem brasileira. Cadernos Pagu, v. 24, n. 1, p. 105-125, 2005.

MORESCHI, C.; SIQUEIRA, D. F. de; DALCIN, C. B.; GRASEL, J. T.; BACKES, D. $\mathrm{S}$. Homenagem a Florence Nightingale e compromisso com a sustentabilidade ambiental. Rev Baiana Enferm., v. 25, n. 2, p. 203-208, 2011.

MORESCHI, C.; REMPEL, C.; BACKES, D. S. A percepção de docentes de cursos de graduação da área da saúde acerca dos resíduos de serviços de saúde. Revista Baiana de Saúde Pública, v. 38, n. 3, p. 647-64, 2014.

MORESCHI, C.; REMPEL, C.; BACKES, D. S.; SIQUEIRA, D. F.; CARRENO, I.; HENDGES D. J. B. Resíduos de serviços de saúde: percepções de docentes e discentes da área da saúde. J Health Sci Inst., v. 32, n. 4, p. 396-403, 2014a

MORESCHI, C.; REMPEL, C.; BACKES, D. S.; CARRENO, I.; SIQUEIRA, D. F.; MARINA. B. The importance of waste from healthcare services for teachers, students and graduates of the healthcare sector. Rev Gaúcha Enferm, v. 35, n. 2, p. 20-26, 2014b.

OMS - Organización mundial de la salud. PNUD - programa de las naciones unidas para el desarrollo. El camino salutable hacia un mundo sostenible. Genebra: OMS, 1995.

PEREIRA, J. C. R. Análise de Dados Qualitativos: Estratégias Metodológicas para as Ciências da Saúde Humanas e Sociais. 3 ed. São Paulo: Edusp, 1999.

PEREIRA, M. S.; ALVES, S. B.; SOUZA, A. C. S.; TIPPLE, A. F. V.; REZENDE, F. R. de; RODRIGUES, É. G. Gerenciamento de resíduos em unidades não hospitalares de urgência e emergência. Rev Latino-Am Enferm., v. 21, n. 8, p. 259-266, 2013.

RIBEIRO, M. C. S.; BERTOLOZZI, M. R. Reflexões sobre a participação da enfermagem nas questões ecológicas. Rev. Esc. Enferm. USP, v. 36, n. 4, p. 300-308, 2002.

RIBEIRO, M. C. S.; BERTOLOZZI, M. R. A questão ambiental como objeto de atuação da vigilância sanitária: uma análise da inserção das enfermeiras nesse campo. Rev.

Latino-Am. Enfermagem, v. 12, n. 5, p. 736-744, 2004.

RODRIGUES, G. S.; FEIJÓ, A. G. S. Ética e ecologia: fundamentos para um ecoturismo responsável e eficaz. In: VIII CONGRESSO DE ECOLOGIA DO BRASIL, 23 a 28 de Setembro de 2007, Caxambu - MG. p. 124-125, 2007. 